CLAWAR2016: 19th International Conference on Climbing and Walking Robots and the Support Technologies for Mobile Machines, London, UK, 12-14 September 2016, World Scientific Publishing Company, Singapore, ISBN:

978-981-3149-12-0.

\title{
CHAOTIC SPIRAL DYNAMICS OPTIMIZATION ALGORITHM
}

\author{
M. R. HASHIM and M. O. TOKHI \\ Department of Automatic Control \& System Engineering, \\ University of Sheffield, Sheffield, UK
}

\begin{abstract}
This paper introduces a chaotic spiral dynamic algorithm (CSDA) for optimization problems. The algorithm is developed by incorporating the chaotic maps pattern into the original spiral dynamic algorithm (SDA) by replacing the rotational radius in SDA with chaotic map, so as to enhance the search diversity of the algorithm avoid potential trap in local optima. The performance of the algorithm is assessed in comparison to SDA with a set of benchmark functions and PD controller tuning of a single link flexible robot manipulator system (SLFRMS). The results show that the CSDA outperforms the SDA with enhanced search capability and escaping local optima.
\end{abstract}

\section{Introduction}

Metaheuristic approaches have attracted significant attention of researches as sensible solver of optimization problems. The metaheuristic optimization algorithm may be categorized into bio-inspired and nature-inspired types.

A bio-inspired algorithmic is associate based on behavior of living organisms whereas a nature-inspired optimization is based on natural phenomena. Examples of bio-inspired optimization algorithm are genetic algorithm (GA) adopted from the process of genetic evolution, particle swarm optimization that mimics the communication behavior of fish and bird flocking. Artificial bee colony algorithm based on the behavior honey bee foraging the food source [1]. Spiral dynamic algorithm (SDA) is based on the spiral pattern such as in tornados [2] is an the example of natural-inspired optimization algorithms.

Since its introduction, SDA has been applied to solving various real problems. It has however, been noted that the algorithm can easily get trapped in local optima when exposed to solving high dimension problems. There has also been an attempt to resolve this issue in SDA. For example, [3] proposed an adaptive formulation by varying the size of radius and displacement of spiral model in order to resolve this issue.

Chaos has unstable behavior and also has complex and bounded characteristic. It generates deterministic sequence and is very sensitive to the 
initial starting point. Many methods have been reported in the literature on attempts to enhance a metaheuristic algorithm are using chaotic sequence [4]. The reported results show encouraging performances, especially with the optimization algorithm escaping local optima points. In this paper, such a strategy is adopted by introducing chaotic sequence as a variable rotational radius value into SDA to arrive at a new optimization algorithm, referred to as chaotic spiral dynamic algorithm (CSDA).

\section{Development of Chaotic Spiral Dynamic Optimization Algorithm}

\subsection{Spiral Dynamic Optimization Algorithm}

SDA is inspired from spiral phenomena such as tornado or nautilus shell. The control parameters for the algorithm are radius and rotation angle. These are two constant and their values are specified by the user [5]. The search strategy in a meta-heuristics is to devise diversification in early stages of the search and intensification in later stages of the search. In SDA, diversification occurs during the early phase of the search and intensification later near the center of the spiral [5]. Figure 1 shows an example of the spiral trajectory for 50 steps with spiral radius $=0.95$ and rotational angle $=\pi / 4$.
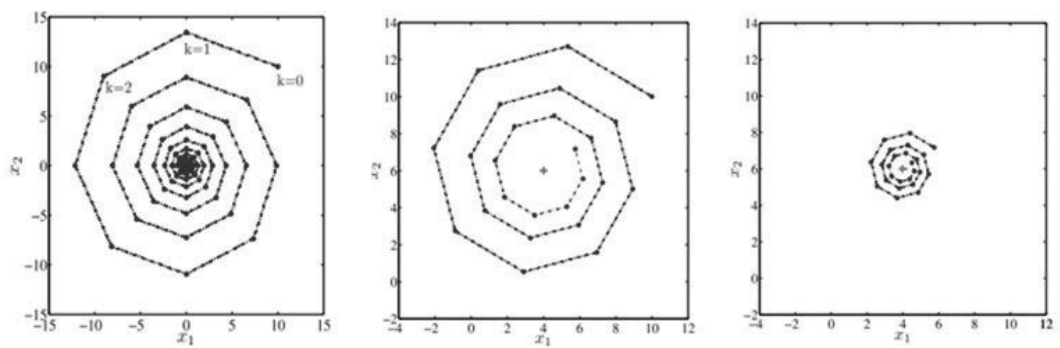

Figure 1. Spiral trajectory [5].

The rotational model of SDA is shown as follows:

$$
x_{i}(k+1)=S_{n}(r, \theta) x_{i}(k)-\left[S_{n}(r, \theta)-I_{n}\right] x^{*}
$$

where $x^{*}$ is the spiral center point, $S_{n}(r, \theta) x_{i}$ is a stable matrix, $x_{i}$ is location of a point at $i=1,2 \ldots, k, r$ is a rotational radius where the value of $r$ is $0<r<$ 1 and $k$ is cycle numbers.

\subsection{Chaotic Maps}

In chaotic theory, the small changes in initial value will have large impact on the final results of the system where this behavior can increase the performance of 
the system [6]. The chaotic map is bounded nonlinear has unstable and ergodic behavior. In this paper, several well-known chaotic maps such as logistic map, Chebyshev maps and Iterative map are used to replace the rotational radius, $r$ in SDA. Mathematical expressions and associated ranges of these maps used in this work are shown in Table 1.

Table 1. Chaotic Maps mathematical expression

\begin{tabular}{|c|c|c|}
\hline Chaotic map & Mathematical expression & Range \\
\hline Chebyshev & $x_{i+1}=\cos \left(i \cos ^{-1}\left(x_{i}\right)\right)$ & $(-1,1)$ \\
\hline logistic & $x_{i+1}=a x_{i}\left(1-x_{i}\right), a=4$ & $(0,1)$ \\
\hline Iterative & $x_{i+1}=\sin \left(\frac{a x}{x_{i}}\right), a=0.7$ & $(-1,1)$ \\
\hline
\end{tabular}

\subsection{Chaotic Spiral Dynamic Optimization Algorithm}

To improve the performance of SDA and also to overcome the problem of algorithm getting trapped at local optima during the search process, the chaotic maps were introduced into SDA by replacing the rotational radius with the chaotic sequence. By adding chaotic sequence in SDA, the SDA step size will become more dynamic and this will help the algorithm come out of local optima. A pseudo-code of the proposed algorithm is shown as follows

Step 1: Initialization.

- $\quad$ Set number of search points, $m$ where $m \geq 2$

- Set rotational angle, $0 \leq \theta \leq 2 \pi$

- $\quad$ Replace rotational radius, $r$ with chaotic sequence, $r_{c h a o}$ where $r_{c h a o}$ is from 0 to 1

- Maximum iteration number $k_{\max }$

- Initial points $x_{i}(0) \in R^{n}, i=1,2, \ldots, m$, randomly placed in the feasible region and choose the centre of spiral $x^{*}=x_{i g}(0), i_{g}=$ $\arg \min f\left(x_{i}(0)\right), i=1,2, \ldots, m$.

Step 2: Update next search point, $x_{i}$.

$$
x_{i}(k+1)=S_{n}\left(r_{\text {chao }}, \theta\right) x_{i}(k)-\left[S_{n}\left(r_{\text {chao }}, \theta\right)-I_{n}\right] x^{*}, i=1,2, \ldots \ldots, m .
$$

Step 3: Update centre point of spiral, $x^{*}$.

$$
x^{*}=x_{i g}(k+1), i_{g}=\arg \min f\left(x_{i}(k+1)\right), i=1,2, \ldots, m .
$$


Step 4: Check whether to terminate the search when $k$ reaches maximum, terminate the search process, otherwise increase $k$ by 1 and go back to step 2 .

\section{Validation using benchmark functions}

The performance and accuracy of the proposed optimization algorithm were assessed using benchmark functions of multimodal and unimodal types. Three different benchmark functions detailed in Table 2 were used in this work.

Table 2. Benchmark test functions.

\begin{tabular}{|c|c|c|}
\hline Objective function $\mathrm{f}(\mathrm{x})$ & Range & $\begin{array}{c}\text { Optimal } \\
\text { solution } \mathrm{f}\left(\mathrm{x}^{*}\right)\end{array}$ \\
\hline$F 1(x)=\sum_{i=1}^{n} x_{i}{ }^{2}$ & {$[-5.12,5.12]$} & 0 \\
\hline$F 2(x)=-20 \exp \left(-0.2 \sqrt{\frac{1}{n} \sum_{i=1}^{n} x_{i}{ }^{2}}\right)$ & {$[-15,30]$} & 0 \\
$-\exp \left(\frac{1}{n} \sqrt{\sum_{i=1}^{n} \cos \left(2 \pi x_{i}\right)}\right)+20+e$ & & \\
\hline$F 3(x)=\frac{1}{4000} \sum_{i=1}^{n} x_{i}{ }^{2}-\prod_{i=1}^{n} \cos \left(\frac{x_{i}}{\sqrt{i}}\right)+1$ & {$[-600,600]$} & 0 \\
\hline
\end{tabular}

In this simulation, the initial parameters used for both SDA and CSDA were $m=40, \theta=\pi / 4, D=30$, maximum iteration $=300$. The simulation results are shown in Table 3 . Each simulation was repeated 30 times and the best average values are indicated in bold.

Table 3. Statistical performance results of the SDA and CSDA.

\begin{tabular}{|c|c|c|c|c|c|c|c|c|}
\hline \multirow{2}{*}{$\begin{array}{c}\text { Benchmark } \\
\text { function }\end{array}$} & \multicolumn{2}{|c|}{ SDA } & \multicolumn{2}{c|}{ CSDA-Logistic } & \multicolumn{2}{c|}{ CSDA-Iterative } & \multicolumn{2}{c|}{ CSDA-Chebyshev } \\
\cline { 2 - 9 } & $\mathrm{Ave}^{\mathrm{a}}$ & $\mathrm{Std}^{\mathrm{b}}$ & $\mathrm{Ave}^{\mathrm{a}}$ & Std $^{\mathrm{b}}$ & $\mathrm{Ave}^{\mathrm{a}}$ & Std $^{\mathrm{b}}$ & Ave $^{\mathrm{a}}$ & Std $^{\mathrm{b}}$ \\
\hline $\mathrm{F} 1$ & 150.41 & 51.72 & $\mathbf{4 9 . 3 8}$ & 26.77 & 68.70 & 33.88 & 49.80 & 42.83 \\
\hline $\mathrm{F} 2$ & 18.93 & 1.18 & 17.78 & 1.25 & 17.99 & 0.62 & $\mathbf{1 7 . 6 5}$ & 1.49 \\
\hline F3 & 511.20 & 166.35 & $\mathbf{1 6 8 . 0 4}$ & 104.23 & 240.52 & 116.47 & 195.55 & 148.73 \\
\hline
\end{tabular}

${ }^{\mathrm{a}}$ Average, ${ }^{\mathrm{b}}$ Standard deviation. 


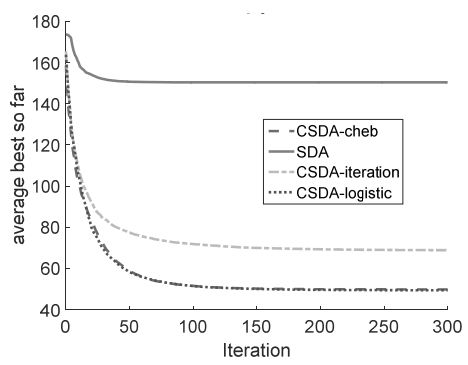

(a) F1

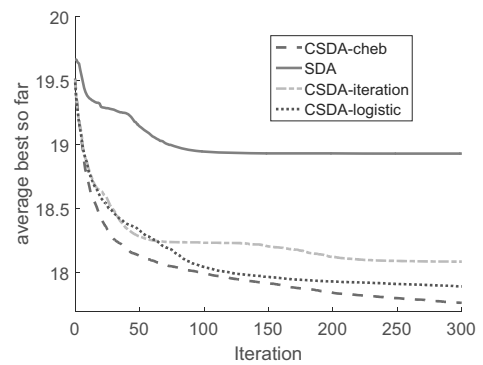

(b) F2

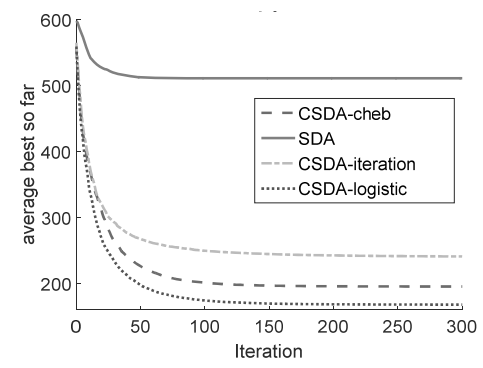

(c) F3

Figure 2. Convergence plots of SDA and CSDA for benchmark functions.

It is noted in Table that the CSDA-Logistic has better results for F1 and F3 and CSDA-Chebyshev outperformed other algorithms in case of F2. It further clear in Figure 2 that SDA converged pre-maturely by getting stuck at local optimum in all three cases whereas the CSDA algorithm continued searching and converged faster and closer to the local optimum points.

\section{SLFMS control tuned using CSDA}

This section presents results of optimization of PD controller design for setpoint tracking of a single link robot manipulator system (SLFRMS) using CSDA. Figure 3, shows a schematic diagram of the SLFRMS.

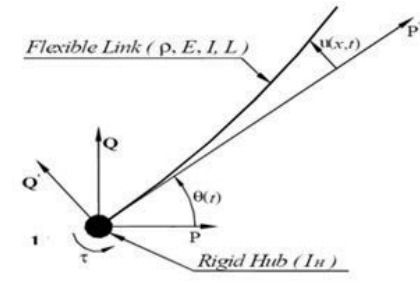

Figure 3. SLMFS schematic diagram [7]. 
The research focus on the optimization of the PD controller using CSDA and the details of the SLMFS can be found in [8] and PD controller structure for SLMFS can be found in [7]. Figure 4 shows the PD control structure of the single link manipulator system, where $\mathrm{K}_{\mathrm{p}}$ and $\mathrm{K}_{\mathrm{v}}$ are the proportional and derivative gains, $A_{c}$ is the manipulator motor drive amplifier gain, and $R_{f}$ is the demanded reference angular position.

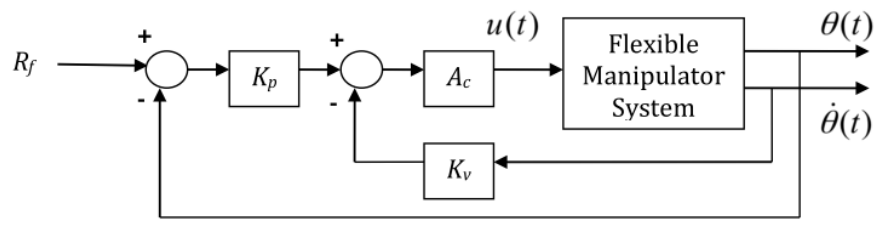

Figure 4. PD controller system for SLFRMS.

CSDA is design to tune the values of $K_{p}$ and $K_{v}$ until it reach the optimum value. There are several of objective functions that may be considered in the optimization algorithm for tuning these parameters such as mean squared error (MSE), integral of squared error (ISE) and integral absolute error (IAE). In this paper, MSE of the angular position is used. To suppressed the response overshoot and undershoot, the absolute maximum overshoot (OS) and undershoot $(U S)$ values are weighted, $w$ and added to the MSE function. Thus the resulting objective is defined as:

$$
J=\left(\frac{1}{N} \sum_{k=1}^{N}(e(t))^{2}\right)+|O S| * w_{1}+|U S| * w_{2}
$$

where, the error $e(t)$ is the difference between reference angular position Rf and actual angular position $\theta(t)$.

The algorithm parameters used in this work to optimize the PD controller were number of searching point, $m=40$, rotational angle, $\theta=\pi / 4$, spiral radius, $r=0.9$, starting point for all chaotic maps was 0.7 . Figure 5 shows hub-angle response of the manipulator with $\mathrm{PD}$ controllers designed using the SDA and the CSDA. It is noted the system response with SDA-designed controller had an overshoot, whereas responses with CSDA-designed controllers did not have overshoot. The rise times of the responses with CSDA-designed controllers were comparable with those of SDA-designed controllers. 


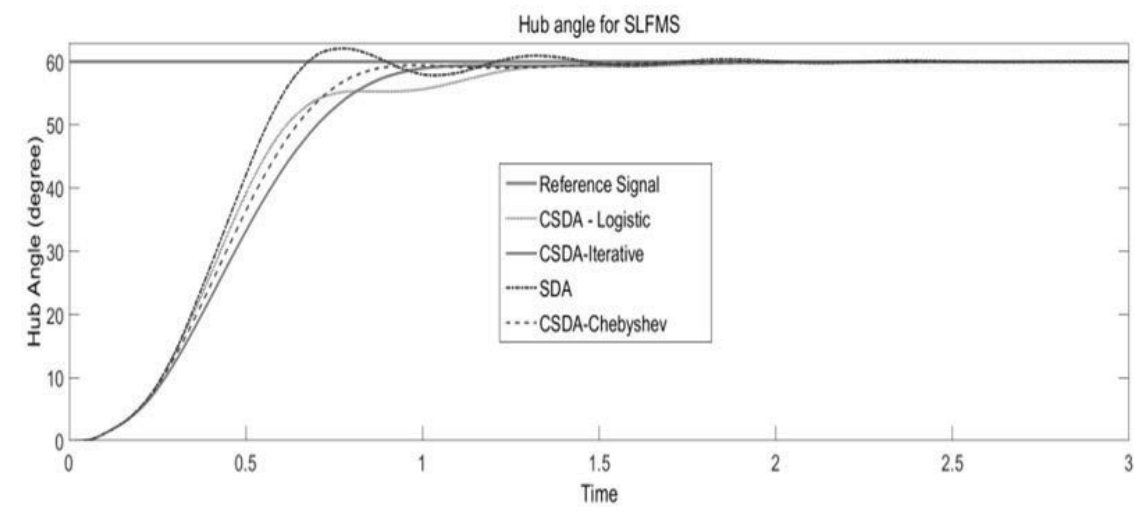

Figure 5. Hub angle output of the SLFMS.

Among the three CSDA-designed control systems, the response with CSDA-iterative type was slightly slower compared to others and the response with CSDA-Chebyshev was the fastest. Numerical values of optimised controller parameters and corresponding system response indicator are shown in Table 4. It is noted that all system designs achieve zero state error, the CSDAdesign system responses reached the set point without overshoot and there was about $4.57 \%$ overshoot in the response of the CSDA-design control system. Furthermore, the response rise times were comparable to one another among the systems and the response settling time of the CSDA-Chebyshev based system was the shortest. Thus, the results above clearly demonstrate the potential benefit of CSDA for solving optimisation problems.

Table 4. Controller and system response parameters.

\begin{tabular}{|c|c|c|c|c|}
\cline { 2 - 5 } \multicolumn{1}{c|}{} & \multicolumn{4}{c|}{ Tuning technique } \\
\hline Measurement & SDA & CSDA-Logistic & CSDA-Iterative & CSDA-Chebyshev \\
\hline $\mathrm{Kp}$ & 3.64 & 2.20 & 0.98 & 1.24 \\
\hline $\mathrm{Kd}$ & 1.13 & 0.83 & 0.45 & 0.52 \\
\hline Overshoot & $4.67 \%$ & 0 & 0 & 0 \\
\hline Settling time & 1.14 & 1.26 & 0.98 & 0.86 \\
\hline Rise time & 0.59 & 0.71 & 0.78 & 0.70 \\
\hline Steady state error & 0 & 0 & 0 & 0 \\
\hline
\end{tabular}

\section{Conclusion}

A chaotic spiral dynamic optimization algorithm by incorporating the potential advantages of chaotic maps into the spiral dynamic algorithm has been developed. The algorithm has capability of jumping out of local optima points and fast convergence to accurate results close to the global optimum. 
Comparative assessments of performance of the proposed algorithm in benchmark test function and controller design of flexible robot manipulator system have shown that it outperforms the original spiral dynamic algorithm and thus can be used as potential contender in solving optimization problems of various kinds.

\section{Acknowledgment}

Mohd Ruzaini bin Hashim is currently on study leave and is sponsored by ministry of higher education Malaysia and Universiti Teknikal Malaysia Melaka.

\section{References}

[1] D. Karaboga, B. Gorkemli, C. Ozturk, and N. Karaboga, "A comprehensive survey: artificial bee colony (ABC) algorithm and applications," Artif. Intell. Rev., vol. 42, no. 1, pp. 21-57, Mar. 2012.

[2] K. Tamura and K. Yasuda, "Spiral Multipoint Search for Global Optimization," 2011 10th Int. Conf. Mach. Learn. Appl. Work., vol. 2, no. 2, pp. 470-475, 2011.

[3] A. N. K. Nasir, M. O. Tokhi, O. Sayidmarie, and R. M. T. Raja IsmaiL, “A novel adaptive spiral dynamic algorithm for global optimization," 2013 13th UK Work. Comput. Intell., pp. 334-341, Sep. 2013.

[4] L. Ding, H. Wu, and Y. Yao, "Chaotic Artificial Bee Colony Algorithm for System Identification of a Small-Scale Unmanned Helicopter," vol. 2015, 2015.

[5] K. Tamura and K. Yasuda, "Spiral Optimization,” no. 1, pp. 1759-1764, 2011.

[6] E. N. Lorenz, "Deterministic Nonperiodic Flow," Journal of the Atmospheric Sciences, vol. 20, no. 2. pp. 130-141, 1963.

[7] M. O. Tokhi, Z. Mohamed, J. M. Martins, M. a. Botto, and J. Sá da Costa, "Approaches for dynamic modelling of flexible manipulator systems," IEE Proc. - Control Theory Appl., vol. 150, no. 4, pp. 401-411, Jul. 2003.

[8] H. Poerwanto, "Dynamic simulation and control of flexible manipulator systems." University of Sheffield, 1998. 